\title{
Fuzzy Smokers Growth Model
}

\author{
Herlinda Nur'Afwa Sofhya ${ }^{1^{*}}$ \\ 1. IAIN Syekh Nurjati Cirebon, west java, Indonesia \\ "Corresponding author: Sunyaragi , Cirebon, West Java, 45132, Indonesia. e-mail addresses: herlindanurafwa15@gmail.com
}

\begin{tabular}{l}
\hline $\mathbf{a} \mathbf{i} \mathbf{i} \mathbf{i} \mathbf{i} \mathbf{f}$ o \\
\hline How to cite this article: \\
Sofhya, H., N. (2020). Fuzzy Smokers Growth \\
Model. Eduma : Mathematics Education Learning \\
And Teaching, 9(1), 57 - 65. \\
doi:http://dx.doi.org/10.24235/eduma.v9i2.7345
\end{tabular}

Article history:

Received: 11 09, 2020

Accepted: 1120,2020

Published: 11, 2020
Copyright (C) 2020

EduMa: Mathematics Education Learning and Teaching under the Creative Commons Attribution 4.0 International License.

\begin{abstract}
a b s t r a c t
FUZZY SMOKERS GROWTH MODEL. The tobacco epidemic is one of the biggest public health problems in the world. Based on the data from WHO, Tobacco kills nearly 6 million people a year around the world. Monitoring tracks of smokers' growth population can be important things for the government to find the best implement policies to overcome this problem. This paper presents a smoker's growth model with uncertainty in the transmission and recovery rate. In classical smoker's growth model, the transmission and recovery rate assumed to be constant. However, in reality, the age of the population is heterogeneous and the transmission among the population may depend on the age of the smoker. Therefore, in this paper, the transmission and recovery rate of smokers' growth model depends on the age of smokers. We divide the transmission and recovery rate into three categories based on age: Children (010), Adolescent (10-30), Adult (30-60). The uncertainty of transmission and recovery rate in this model represented by a triangular fuzzy number. The most important things in the model are the basic reproduction number. A basic reproduction number is an indicator of when the endemic case will occur. Therefore, the main focus of this paper is to determine the basic reproduction number of fuzzy smokers growth models using the fuzzy expected value concept. The result is the basic reproduction number of the fuzzy model is an interval. This may can be used as an upper and lower limit of the basic reproduction number.
\end{abstract}

\section{Key word s :}

Fuzzy Smokers Growth Model, Fuzzy Expected Value, Basic Reproduction Number 


\section{INTRODUCTION}

Tobacco epidemic is one of the longest and the biggest public health problem in the world. Tobacco killing nearly 6 million people a year around the world (WHO, 2019). Many countries still struggling to over come this problem. Monitoring tracks of smokers growth population can be important things for goverment to find best implement policies to overcome this problem. There are many ways to monitoring tracks of smokers growth population, one of which using a mathematical model simulation.

In 1997, Castillo-Garsow et al. present a simple mathematics model PQS for smokers growth population. In PQS model the population divided into three categories: potential smokers $(\mathrm{P})$, Quitted smokers (Q) and smokers (S). Then in 2009 Gunawan, A. Y and Nurtaman, M. E construct smokers growth model adopted from SIR model. Bassically the two smokers growth models almost the same but both have different model analyzes. The classical smokers growth model in this paper will be adapted from those two models by adding the mortality rate for each variable. This classical model will be modified into a fuzzy smokers growth model.

In the classical model the transmission among the population is usually assumed to be constant. However in the fact the transmission and recovery rate among the population are uncertain. Stochastic models with probability distributions are usually used to model the phenomena that contain an untcertainty variable. However, uncertain variables can also be described as fuzzy sets. In 2003, Barros et al presented uncertainty variables of transmission as a fuzzy sets on a simple SI model. This method is very interesting to be adapted and developed in the smokers growth model. This is due to the fact that the age of individuals in the population is heterogeneous. So the transmission and recovery in the population is uncertain and may depend on age. As we know that in the fact children less interested in smoking than adolescent and adults. Therefore, the transmission rate in children category must be different from the transmission rate in adolescent and adult category. Likewise with recovery rate in the population. Adult category will be more difficult to recover from cigarette addiction than the adolescent and children category, because they have been smoking for a long time. This phenomenon is very suitable if we describe it as a fuzzy sets which has a membership function. Therefore, this paper will present a fuzzy model with the transmission and recovery rate are not constant but contains an uncertainty and depending on age. The transmission and recovery rate will be described as a triangular fuzzy numbers and will be devided into three catagories based on age. The main focus on this paper is calculating a basic reproduction numbers of the fuzzy model. Basic reproduction numbers is one of the important things of the model. Using basic reproduction numbers, we can calculate when the endemic case will occur. Calculations involving fuzzy sets are different from calculations in classical model because there are uncertain variables. A fuzzy expected value method is needed to obtain the expected value of basic reproduction number in fuzzy model.

\section{LITERATURE REVIEW}

\section{Fuzzy sets}

Fuzzy sets is often used to represent an uncertainty. The basic idea of a fuzzy sets concerns of the flexibility. Zadeh is the fisrt who introduce fuzzy sets in 1965 . The fuzzy set consists in relaxing the belongingness constraint required by the function above, assuming intermediate membership 
values into the unit interval $[0,1]$. The membership values can be understood as the degrees to which each object is compatible with the properties or features that characterizes the group. There are several kinds of membership functions for fuzzy sets, some of which are commonly used are triangular fuzzy number. A fuzzy set is called triangular fuzzy number if the membership value can be represented by a triangular function. This function is specified by three parameters, $\mathrm{F}(x: a, b, c)$ such as:

$$
F(x: a, b, c)=\left\{\begin{array}{cc}
\frac{x-a}{b-a} & a \leq x<b \\
\frac{c-x}{c-b} & b \leq x<c \\
0 & x>c
\end{array}\right.
$$

Where $b$ is the central value.

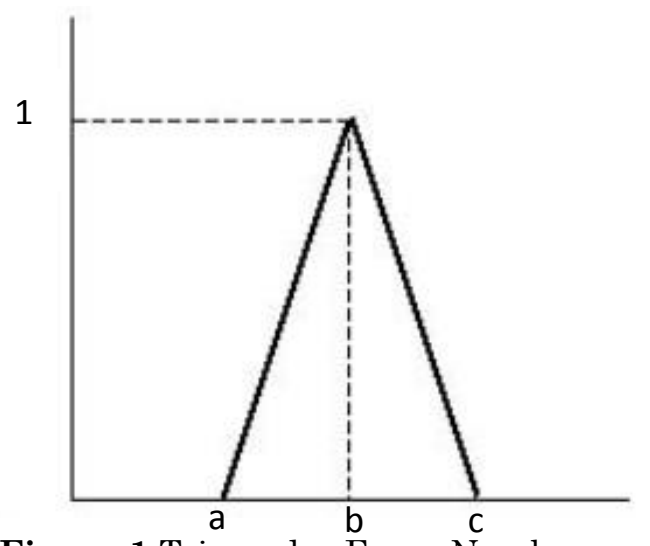

Figure 1 Triangular Fuzzy Number

\section{Fuzzy Measure}

To obtain Fuzzy Expected Value of an uncertain variable described as a fuzzy numbers, we need a fuzzy measure. There are many definition of fuzzy measure. In this paper we use fuzzy measure adopted from sugeno measure, which define as:

A mapping $\mu: P(\Omega) \rightarrow[0,1]$, is called a fuzzy measure if,

1. $\mu(\phi)=0$ and $\mu(\Omega)=1$;

2. $\mu(A) \leq \mu(B)$ always that $A \subseteq B$.

Under this definition the sugeno measure is a particular case of the fuzzy measure (Massad et al, 2008).

\section{Fuzzy Expected Value (FEV)}

FEV is a method used to get the expected value of an uncertain variable described as a fuzzy numbers. FEV is different from a method to get the expected value of a random variable which contains uncertainty in the probability theory. The difference of the two methods is the integral used to obtain the expected value of a variabel. The integral of lebesque is used to obtain the expected value of a random variable with regard to probability measure. On the other hand, the integral of Sugeno is used to obtain the expected value of an uncertain variable with regard to fuzzy measure. FEV define as:

Let $X: \Omega \rightarrow[0,1]$ be an uncertain variable (typically a membership function) and $\mu$ a fuzzy measure on $\Omega$. The Fuzzy Expected Value (FEV) of $X$ is the real number:

$$
F E V(X)=(S) \int_{\Omega} X d \mu=\operatorname{Sup}_{0 \leq \alpha \leq 1}\{\min [\alpha, F(\alpha)]\}
$$

Where $F(\alpha)=\mu\{\omega \in \Omega: X(\omega) \geq \alpha\}$ (Massad et al, 2008).

\section{METHODS}

Smokers growth model usually represented as a deterministic model. The output of deterministic model can calculated exactly without involvement the randomness. But in reality there are an uncertainty. Therefore, the first step of this paper will present a simple deterministic model to describe smokers growth phenomenon. The population in this model assumed to be constant. It means the birth and death rate are equal. In this paper, we divided the population into three sub population: Active Smokers, Potential Smokers, Quitted Smokers. An active smoker is a person who active smoking at least one cigarette a day. A potential smoker is a person who potentially become an active smoker in the future. and quitted smoker is a person who has successfully quit from smoking addiction. This deterministic model we called classical model. In this classical model the transmission and recovery among the population assumed to be constant. However in reality, There are an uncertainty, the age of population are heterogeneous. So the transmission and recovery rate among the population are not constant but may depend on age. 
Therefore, on the next step we modify the classical model to be a new model that contains the uncertainty. We derived the uncertainty as a fuzzy sets. So we called this a new model as a fuzzy model. The transmission among the populasi in fuzzy model is not constant but depend on age. The population in fuzzy model will divided into three categories based on age. According to WHO, the age groupings of population can be divided into three categories as follows: children (0-10) years old, adolecent (10-30) years old, adult (30$60)$ years old. As we often see the phenomenon in reality that children are usually not interested in cigarettes. That's why in fuzzy model, the transmission rate from Potential Smokers to Active Smokers in children categories is less than the transmission rate in adolecent or adult categories. We also often see the phenomenon in reality that adult categories more difficult to recovery from smoking addiction because they have been smokers for along time. Therefore, in fuzzy model the recovery rate from Active Smokers to become Quitted Smokers in adult categories is less than the recovery rate in adolecent or adult categories. In this fuzzy model, the uncertainty of transmission and recovery among the heterogeneity of population ages will represented as a fuzzy numbers. Therefore this model called as a fuzzy smokers growth model.

The main focus on this paper is find out the basic reproduction number of model. The method to obtain basic reproduction number of fuzzy model is different from the method to obtain basic reproduction number in classical model. In classical model which a deterministic model, we can directly determining the basic reproduction number of model. However, in fuzzy model which contains the uncertainty we need FEV method to obtain the expectation value of an uncertain variable with regard to fuzzy measure. So, in this paper we use FEV method to obtain basic reproduction number of fuzzy model. Then we can see the difference between basic reproduction number in classical model and the basic reproduction number in fuzzy model.

\section{RESULT AND DISCUSSION}

\section{Classical smokers growth model}

A simple model to describe smokers growth phenomenon with transmission and recovery among the population can be described by compartment diagram in Figure 3,

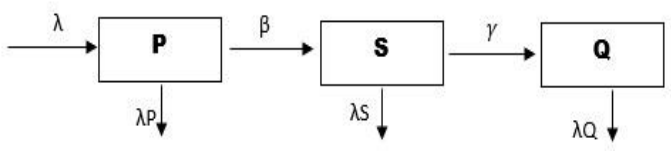

Figure 3 Compartment Diagram.

Where $\mathrm{P}$ is proportion of Potential smokers, $\mathrm{S}$ is proportion of Active smokers, and $\mathrm{Q}$ is proportion of Quitted smokers. The population in this model assumed to be constant so death rate $=$ birth rate $=\lambda . \beta$ is the transmission rate from a potential smoker to become an active smoker and $\gamma$ is the recovery rate from an active smokers to become quitted smokers. Transmission and recovery rate in this classic model assumed to be constant. The compartemen diagram in Figure 1 can be mathematically described by deterministic model,

$\frac{d P}{d t}=\lambda-\beta P-\lambda P$

$\frac{d S}{d t}=\beta P-\gamma S-\lambda S$

$\frac{d Q}{d t}=\gamma S-\lambda Q$

There are two equilibrium point from classical smokers growth model: $E_{1}=$ $(1,0,0)$ and $E_{2}=\left(\frac{\lambda+\gamma}{\beta}, \frac{\lambda(\beta-(\lambda+\gamma))}{\beta(\lambda+\gamma)}, \frac{\gamma(\beta-(\lambda+\gamma))}{\beta(\lambda+\gamma)}\right)$. $E_{1}$ is Disease Free Equilibrium (DFE) and $E_{2}$ is Endemic Equilibrium (EE). From the stability analysis of two equilibrium point, we obtain that $E_{1}$ which the DFE stable when $\beta<(\lambda+\gamma)$ and $E_{2}$ which the EE stable when $\beta>(\lambda+\gamma)$. Based on these results, we can contruct $R_{0}=\frac{\beta}{\lambda+\gamma}$. So it fulfills the basic reproduction number 
requirements. The basic reproduction number must fulfill the conditions where $R_{0}<1$, the endemic cases never occur or in a long time the disease will disappear and where $R_{0}>1$, the endemic cases will occur. Therefore the basic reproduction number of this classical model is $R_{0}=\frac{\beta}{\lambda+\gamma}$. In this deterministic model, $\beta, \gamma$, and $\lambda$ are constant. So if we know the value of parameters $\beta, \gamma$, and $\lambda$, we can directly calculate the basic reproduction number of this classical model. The parameters of classical model are the exact number. So the basic reproduction number of classical model is an exact number.

\section{Fuzzy smokers growth model}

Fuzzy smokers growth model is obtained by modifying the classical smokers growth model. In this model, the heterogeneity of population ages is considered. Therefore, the transmission and recovery among the population is not constant but depending on age. Therefore the population divided by three categories based on age. Children, adolecent and adult. The age distribution of each categories will described as a triangular fuzzy number with membership function $f(u)$;

$f(u)=\left\{\begin{array}{cl}0 & , u<\bar{u}-\sigma \\ \frac{1}{\sigma}(u-(\bar{u}-\sigma)) & , \bar{u}-\sigma \leq u \leq \bar{u} \\ \frac{1}{\sigma}((\bar{u}+\sigma)-u) & , \bar{u}<u \leq \bar{u}+\sigma \\ 1 & , u>\bar{u}+\sigma\end{array}\right.$

Where $\bar{u}$ is the central value and $\sigma$ gives the dispersion of papulation ages. Triangular fuzzy number in this fuzzy modal represented by Figure 4 .

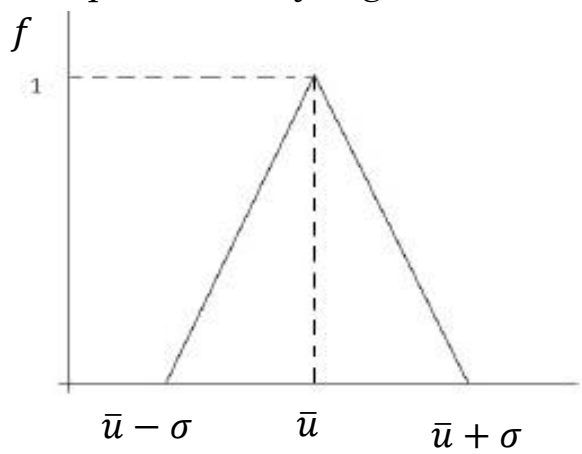

Figure 4 Triangular Fuzzy Number in Fuzzy Model
As we often see in reality that children are usually not interested in cigarettes. It means the transmission from potential smokers to become active smokers in children categories is less than in adult and adolecent categories. In this model, we derived the transmission rate as a fuzzy sets with membership function $\beta(u)$;

$$
\beta(u)=\left\{\begin{array}{cl}
0 & , 0<u \leq 10 \\
\frac{u-10}{20} & , 10<u \leq 30 \\
1 & , u>30
\end{array}\right.
$$

where $u$ is an age of individu in population. The transmission rate can represented by Figure 5.

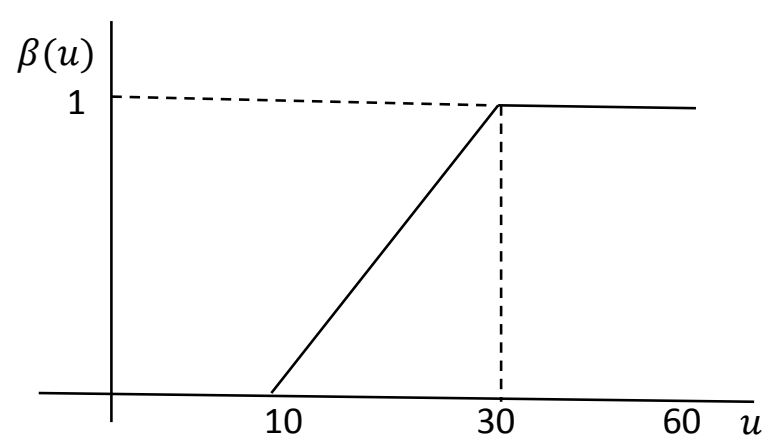

Figure 5 Transmission Rate

In reality, we often see that people in adult categories usually difficult to quit from addiction of cigarette because they have been smokers for along time. So that the recovery rate in adult categories is less than in children and adolecent categories. Especially for people over 50 years old, it will be very difficult to recover from smoking addiction. So in this model we assume the recovery rate for people over 50 years old is minimun. Therefore, in this model we derived recovery rate as a fuzzy sets with membership function $\gamma(u)$;

$$
\gamma(u)= \begin{cases}\frac{\left(\gamma_{\min }-1\right) u}{50}+1, & , u \leq 50 \\ \gamma_{\min } & , u>50\end{cases}
$$

with $\mathrm{u}$ is age and $\gamma_{\min }$ is recovery rate minimum for people over 50 years old. The recovery rate can be represented by Figure 6. 


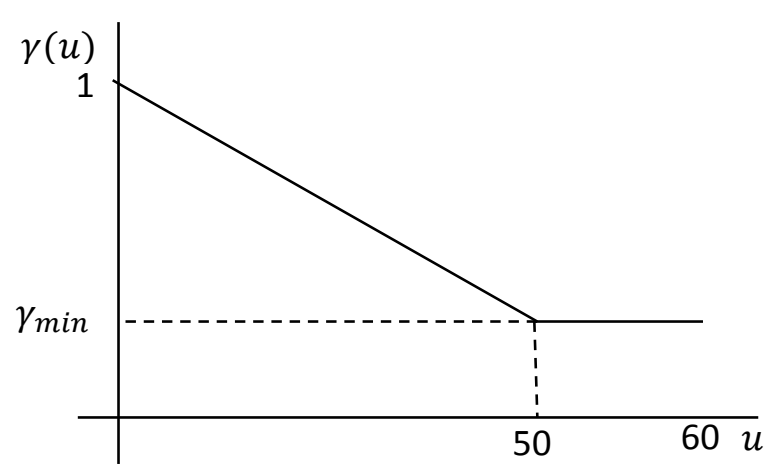

Figure 6 Recovery Rate

The heterogeneity of population ages with transmission and recovery rate depend on age in this fuzzy model can be described by Figure 7.

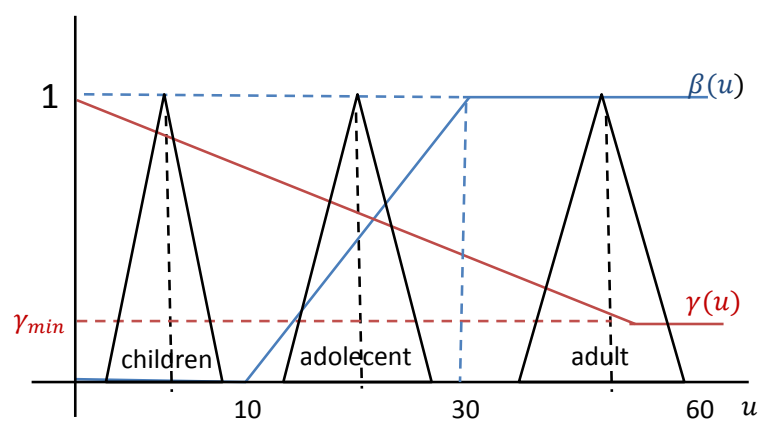

Figure 7 Heterogeneity of Population Ages

Basicly, fuzzy smokers growth model and classical smokers growth model are similar. The difference between the two is in the classical model, age of population assumed homogeneous. But in fuzzy model, age of population is heterogeneous. So there are an uncertainty of transmission and recovery rate among the population in fuzzy model. the transmission and recovery rate in this fuzzy model are not constant but depend on age.In fuzzy model the transmission and recovery rate defined by $\beta(u)$ and $\gamma(u)$. Adopted from classical model, we obtain fuzzy smokers growth model;

$$
\begin{aligned}
& \frac{d P}{d t}=\lambda-\beta(u) P-\lambda P \\
& \frac{d S}{d t}=\beta(u) P-\gamma(u) S-\lambda S \\
& \frac{d Q}{d t}=\gamma(u) S-\lambda Q
\end{aligned}
$$

If we look at the model equation there is not much difference between the fuzzy model and the classical model. But it will much different in calculation because in fuzzy model there is an uncertainty variable that regard to fuzzy measure. Different from the calculation in classical model which can be calculated directly, in fuzzy model we need FEV method to obtain the expected value of an uncertain variable that regard to fuzzy measure.

Like in classical model, we obtain two equilibrium point in fuzzy model. $E_{1}=$ $(1,0,0)$ as DFE and $E_{2}=$ $\left(\frac{\lambda+\gamma(u)}{\beta(u)}, \frac{\lambda(\beta(u)-(\lambda+\gamma(u)))}{\beta(u)(\lambda+\gamma(u))}, \frac{\gamma(\beta(u)-(\lambda+\gamma(u)))}{\beta(u)(\lambda+\gamma(u))}\right)$ as EE. Adopted from basic reproduction number of classical model we obtain $R_{0}(u)=\frac{\beta(u)}{\gamma(u)+\lambda} \cdot \quad \beta(u)$ and $\gamma(u)$ is a fuzzy number with $u$ described as a triangular fuzzy number. Therefore, to obtain the basic reproduction number of fuzzy model, we have to calculate the expected value of $R_{0}(u)$ which is the fuzzy numbers. As we discussed before, we need FEV method to get an expectation value of uncertain variable represented by fuzzy numbers. Before calculating the FEV of $R_{0}(u)$, we have a problem because $\beta(u)<1, \gamma(u)<1$, and $\lambda<1$ so $R_{0}(u)=\frac{\beta(u)}{\gamma(u)+\lambda}$ can be greater than 1 . This should not happen because if $R_{0}$ greater than 1 , then it is undefined in a fuzzy sets where all members are expressed in range 0 to 1 . we can't get the FEV of $R_{0}(u)$ if $R_{0}$ greater than 1 . So we have to modify $R_{0}(u)$ to be less than 1 .

$\gamma_{\min }<\gamma(u)$

$\gamma_{\min }+\lambda<\gamma(u)+\lambda$

$\frac{\gamma_{\min }+\lambda}{\gamma(u)+\lambda}<1$

As we know before $\beta(u)<1$ so we obtain $\frac{\left(\gamma_{\min }+\lambda\right) \beta(u)}{\gamma(u)+\lambda}<1$. Based on the properties of expected value, the expected value of a constant is the same as that constant. As we know $\left(\gamma_{\min }+\lambda\right)$ is constant, it means the expected value of $\left(\gamma_{\min }+\lambda\right)$ is $\left(\gamma_{\min }+\right.$ $\lambda)$. Therefore, to make FEV of $R_{0}(u)$ is well defined without changing the value of basic 
reproduction number in fuzzy model so we can construct.

$$
\begin{aligned}
& R_{0_{f u z z y}}=\frac{1}{\left(\gamma_{\min }+\lambda\right)} F E V\left[\frac{\left(\gamma_{\min }+\lambda\right) \beta(u)}{\gamma(u)+\lambda}\right] \\
& R_{0 \text { fuzzy }}=\frac{1}{\left(\gamma_{\min }+\lambda\right)} F E V\left[\left(\gamma_{\min }+\lambda\right) R_{0}(u)\right]
\end{aligned}
$$

The result of $R_{0 f u z z y}$ depends on $u$ and $u$ represented as a fuzzy triangular number $f(u)$. To obtain FEV we need to define a fuzzy measure of $u$. Define $\mu\{U\}=\operatorname{Sup}_{u \in U} f(u)$ as a fuzzy measure. We can see from the definition of fuzzy measure that $\mu\{\mathrm{U}\}$ qualifies as a fuzzy measure. By using fuzzy measure $\mu$, we can obtain $\operatorname{FEV}\left[\left(\gamma_{\min }+\lambda\right) R_{0(u)}\right]$.

$$
F E V\left[\left(\gamma_{\min }+\lambda\right) R_{0(u)}\right]=\operatorname{Sup}_{0 \leq \alpha \leq 1}\{\min [\alpha, F(\alpha)]\} .
$$

Where $F(\alpha)=\mu\left\{u:\left(\gamma_{\min }+\lambda\right) R_{0(u)} \geq \alpha\right\}$. As we can see, FEV $\left[\left(\gamma_{\min }+\lambda\right) R_{0(u)}\right]$ depend on $\beta(u)$ and $\gamma(u)$. As we discussed before, in this fuzzy model, the population devided by 3 categories based on population ages and each categories have the differences of transmission and recovery rate. Therefore $R_{0 f u z z y}$ will be calculated from each age category.

\section{a. Children}

The age of the population in children category is $0-10$ years old. In this category $\beta(u)=0$ and $\gamma(u)=\frac{\left(\gamma_{\min }-1\right) u}{50}+1$. So that we can calculate $F E V\left[\left(\gamma_{\min }+\lambda\right) R_{0(u)}\right]$.

$$
\begin{aligned}
& \operatorname{FEV}\left[\left(\gamma_{\min }+\lambda\right) R_{0(u)}\right]=\operatorname{Sup}_{0 \leq \alpha \leq 1}\{\min [\alpha, \mu\{u: 0 \geq \alpha\}]\} \\
&=\operatorname{Sup}_{0 \leq \alpha \leq 1}\{\min [\alpha, \mu\{\phi\}]\} \\
&=\operatorname{Sup}_{0 \leq \alpha \leq 1}\{\min [\alpha, 0]\} \\
&=0 \\
& R_{0 \text { fuzzy }}=\frac{1}{\left(\gamma_{\min }+\lambda\right)} \operatorname{FEV}\left[\left(\gamma_{\min }+\lambda\right) R_{0}(u)\right] \\
&=0
\end{aligned}
$$

In children categories we obtain $R_{0 \text { fuzzy }}=0$. It means in this category the endemic cases never occur and for along time there will no an active smokers in children categories.

\section{b. Adolecent}

The age of the population in adolecent category is 10-30 years old. The transmission and recovery rate in this category are $\quad \beta(u)=\frac{u-10}{20}$ and $\gamma(u)=$ $\frac{\left(\gamma_{\min }-1\right) u}{50}+1$. Then we can calculate $\operatorname{FEV}\left[\left(\gamma_{\min }+\lambda\right) R_{0(u)}\right]$.

$$
F E V\left[\left(\gamma_{\min }+\lambda\right) R_{0(u)}\right]=\operatorname{Sup}_{0 \leq \alpha \leq 1}\{\min [\alpha, F(\alpha)]\}
$$

Where $F(\alpha)=\mu\left\{u: \frac{\left(\gamma_{\min }+\lambda\right) \beta(u)}{\gamma(u)+\lambda} \geq \alpha\right\}$. With direct calculation, we obtain $\mathrm{F}(\alpha)=\mu(\Omega)=$ 1 when $\alpha=0, \mathrm{~F}(\alpha)=\mu(\phi)=0$ when $\alpha=1$, $\mathrm{F}(\alpha)=\mu\left[u_{\alpha}, 30\right]$ when $0<\alpha<1$, with $u_{\alpha}$ is when $\frac{\left(\gamma_{\min }+\lambda\right) \beta(u)}{\gamma(u)+\lambda}=\alpha$. For $0<\alpha<1$ we divide $F(\alpha)$ into 3 cases. When $0<\alpha \leq$ $\frac{\left(\gamma_{\min }+\lambda\right) \beta(\bar{u})}{\gamma(\bar{u})+\lambda}$ we obtain $F(\alpha)=1$, when $\frac{\left(\gamma_{\min }+\lambda\right) \beta(\bar{u})}{\gamma(\bar{u})+\lambda} \leq \alpha \leq \frac{\left(\gamma_{\min }+\lambda\right) \beta(\bar{u}+\sigma)}{\gamma(\bar{u}+\sigma)+\lambda}$ we obtain $F(\alpha)=f\left(u_{\alpha}\right)$, and when $\frac{\left(\gamma_{\min }+\lambda\right) \beta(\bar{u}+\sigma)}{\gamma(\bar{u}+\sigma)+\lambda}<$ $\alpha<1$ we obtain $F(\alpha)=0$. Therefore we get $F(\alpha)$ for $0 \leq \alpha \leq 1$.

$F(\alpha)=\left\{\begin{array}{rrr}1, & 0 \leq \alpha \leq \frac{\left(\gamma_{\min }+\lambda\right) \beta(\bar{u})}{\gamma(\bar{u})+\lambda} \\ f\left(u_{\alpha}\right), & \frac{\left(\gamma_{\min }+\lambda\right) \beta(\bar{u})}{\gamma(\bar{u})+\lambda} \leq \alpha \leq \frac{\left(\gamma_{\min }+\lambda\right) \beta(\bar{u}+\sigma)}{\gamma(\bar{u}+\sigma)+\lambda} \\ 0, & \frac{\left(\gamma_{\min }+\lambda\right) \beta(\bar{u}+\sigma)}{\gamma(\bar{u}+\sigma)+\lambda}<\alpha \leq 1\end{array}\right.$

As we see the function of $\mathrm{F}$ is decreasing function so with direct calculation we can obtain FEV $\left[\left(\gamma_{\min }+\lambda\right) R_{0(u)}\right]$

$$
\begin{aligned}
\frac{\left(\gamma_{\min }+\lambda\right) \beta(\bar{u})}{\gamma(\bar{u})+\lambda} \leq & \operatorname{FEV}\left[\left(\gamma_{\min }+\lambda\right) R_{0(u)}\right] \\
& \leq \frac{\left(\gamma_{\min }+\lambda\right) \beta(\bar{u}+\sigma)}{\gamma(\bar{u}+\sigma)+\lambda}
\end{aligned}
$$

And we obtain $R_{0}$ fuzzy

$$
\begin{gathered}
\frac{\beta(\bar{u})}{\gamma(\bar{u})+\lambda} \leq \frac{1}{\gamma_{\min }+\lambda} \operatorname{FEV}\left[\left(\gamma_{\min }+\lambda\right) R_{0(u)}\right] \leq \frac{\beta(\bar{u}+\sigma)}{\gamma(\bar{u}+\sigma)+\lambda} \\
R_{0}(\bar{u}) \leq R_{0}{ }_{f u z z y} \leq R_{0}(\bar{u}+\sigma)
\end{gathered}
$$

The value of $\bar{u}$ in this category is $\bar{u}=20$ so we obtain interval of basic reproduction number for adolecent categories.

$$
R_{0}(20) \leq R_{0 \text { fuzzy }} \leq R_{0}(20+\sigma)
$$




\section{c. Adult}

The age of the population in Adult category is 30-60 years old. The transmission and recovery rate in this categories are $\beta(u)=1$ and for $30 \leq u<50 \quad \gamma(u)=$ $\frac{\left(\gamma_{\min }-1\right) u}{50}+1$, for $u \geq 50 \quad \gamma(u)=\gamma_{\text {min }}$. So that we can calculate $F E V\left[\left(\gamma_{\min }+\lambda\right) R_{0(u)}\right]$.

$$
F E V\left[\left(\gamma_{\min }+\lambda\right) R_{0(u)}\right]=\operatorname{Sup}_{0 \leq \alpha \leq 1}\{\min [\alpha, F(\alpha)]\}
$$

Where $F(\alpha)=\mu\left\{u: \frac{\left(\gamma_{\min }+\lambda\right) 1}{\gamma(u)+\lambda} \geq \alpha\right\}$. Through difficult calculation we obtain when $\alpha=0$ we obtain $F(\alpha)=\mu(\Omega)=1$. When $0<\alpha \leq$ $\frac{\left(\gamma_{\min }+\lambda\right)}{\gamma(\bar{u})+\lambda}$ we obtain $F(\alpha)=1$. When $\alpha=1$ we obtain $F(\alpha)=\mu(\phi)=0$. When $0<\alpha<1$ we obtain $F(\alpha)=\mu\left[u_{\alpha_{1}}, 60\right]$ with $u_{\alpha_{1}}=$ $\left\{u: \frac{\left(\gamma_{\min }+\lambda\right)}{\gamma(u)+\lambda}=\alpha\right\}$. Then for $0<\alpha<1$ we divide $F(\alpha)$ into 3 cases. When $0<\alpha \leq$ $\frac{\left(\gamma_{\min }+\lambda\right)}{\gamma(\bar{u})+\lambda} \quad$ we obtain $\quad F(\alpha)=1$, when $\frac{\left(\gamma_{\min }+\lambda\right)}{\gamma(\bar{u})+\lambda}<\alpha \leq \frac{\left(\gamma_{\min }+\lambda\right)}{\gamma(\bar{u}+\sigma)+\lambda} \quad$ we obtain $\quad F(\alpha)=$ $f\left(u_{\alpha_{1}}\right)$, and when $\frac{\left(\gamma_{\min }+\lambda\right)}{\gamma(\bar{u}+\sigma)+\lambda}<\alpha<1$ we obtain $F(\alpha)=0$. Thus we get $F(\alpha)$ for $0 \leq$ $\alpha \leq 1$.

$$
F(\alpha)=\left\{\begin{array}{cr}
1, & 0 \leq \alpha \leq \frac{\left(\gamma_{\min }+\lambda\right)}{\gamma(\bar{u})+\lambda} \\
f\left(u_{\alpha_{1}}\right), & \frac{\left(\gamma_{\min }+\lambda\right)}{\gamma(\bar{u})+\lambda} \leq \alpha \leq \frac{\left(\gamma_{\min }+\lambda\right)}{\gamma(\bar{u}+\sigma)+\lambda} \\
0, & \frac{\left(\gamma_{\min }+\lambda\right)}{\gamma(\bar{u}+\sigma)+\lambda}<\alpha \leq 1
\end{array}\right.
$$

As we see the function of $\mathrm{F}$ is decreasing function so with direct calculation we can obtain $\mathrm{FEV}\left[\left(\gamma_{\min }+\lambda\right) R_{0(u)}\right]$

$$
\frac{\left(\gamma_{\min }+\lambda\right)}{\gamma(\bar{u})+\lambda} \leq \operatorname{FEV}\left[\left(\gamma_{\min }+\lambda\right) R_{0(u)}\right] \leq \frac{\left(\gamma_{\min }+\lambda\right)}{\gamma(\bar{u}+\sigma)+\lambda}
$$

Based on the result of FEV $\left[\left(\gamma_{\min }+\lambda\right) R_{0(u)}\right]$ we obtain $R_{0}$ fuzzy .

$$
\begin{gathered}
\frac{1}{\gamma(\bar{u})+\lambda} \leq \frac{1}{\gamma_{\min }+\lambda} \operatorname{FEV}\left[\left(\gamma_{\min }+\lambda\right) R_{0(u)}\right] \leq \frac{1}{\gamma(\bar{u}+\sigma)+\lambda} \\
R_{0}(\bar{u}) \leq R_{0_{f u z z y}} \leq R_{0}(\bar{u}+\sigma)
\end{gathered}
$$

The value of $\bar{u}$ in this category is $\bar{u}=45$ so we obtain range of basic reproduction number for adult category.

$$
R_{0}(45) \leq R_{0}{ }_{f u z z y} \leq R_{0}(45+\sigma)
$$

Then, if we generalize from case b) and c) where there are no age categories in population. we obtain the basic reproduction number of fuzzy model if the transmission and recovery among the population is not zero

$$
R_{0}(\bar{u}) \leq R_{0 \text { fuzzy }} \leq R_{0}(\bar{u}+\sigma) .
$$

Where $\bar{u}$ can be represented as an average age of the population. We can see that basic reproduction number in fuzzy model is different from basic reproduction number in classical model. we obtain the basic reproduction model in fuzzy model is an interval while in the classic model is an exact number. This interval from fuzzy model may can be used as an upper and lower limit of the basic reproduction number of the smokers growth model with $R_{0}(\bar{u})$ as an upper limit and $R_{0}(\bar{u}+\sigma)$ as a lower limit.

Basic reproduction number is very important parameter because it is the indicator when the endemic cases accurs. So we have to be careful to determining basic reproduction number of the model.If we analyze further, In classical model the age of population are assumed to be homogeneous. People usually take the average age of population to determining the parameter in deterministic model. Let $\bar{u}$ is the average age of population. So if we adopted transmision and recovery rate from fuzzy model, we obtain $R_{0}$ of clasic model is $R_{0}(\bar{u})$. As we discussed before that where there are no age category in population, $\bar{u}$ in fuzzy model can be represented as the average age of the population. So if we compare the results of basic reproduction number in classical model and in fuzzy model, we get the relationship between the two

$$
R_{0}(\bar{u}) \leq R_{0}{ }_{f u z z y} .
$$

As we can see basic reproduction number of classical model is less than basic reproduction number of fuzzy model. These result of basic reproduction number in fuzzy model provides a new perspective that maybe so far the basic reproduction number that we have usually calculated in 
classical model is under estimation because in classical model not pay attention to the uncertainty factor of population heterogeneity. If we look at the real phenomenon, the fuzzy smokers growth model can be more realistic than classical smokers growth model because it takes into account the uncertainty factor.

\section{CONCLUSION AND IMPLICATION}

\section{a. Conclusion}

Based on the result and discussion in this study, we get that fuzzy smokers growth model which contains an uncertainty of the transmission and recovery rate gives different result from the calculation in the classical model. In the classical model which is deterministic model we will always obtain the basic reproduction number of the model is an exact number. But if modify the classical model to become a fuzzy model, we obtain the basic reproduction number of the fuzzy model is an interval. This interval may can be used as an upper and lower limit of the basic reproduction number of the smokers growth model. Then, we obtain the relationship between the basic reproduction number of classical smokers growth model and fuzzy smokers growth model. The basic reproduction number of classical model is less than basic reproduction number of fuzzy model. These result provides a new perspective that maybe so far the basic reproduction number that we have usually calculated in the classical model is under estimation because in classical model not pay attention to the uncertainty factor of population heterogeneity.

\section{b. Implication}

If we generalize the result of this study. Maybe we can modify the other classical mathematical model which is deterministic model into a fuzzy model that contains the uncertainty, so the mathematical model can be more realistic. Based on the results of the basic reproduction number in the fuzzy model we can get the interval of basic reproduction number. With this interval we can be more careful and have several alternatives in determining when the endemic case will occur.

\section{REFERENCES}

Barros, L. C., Bassarnezi, R. Z. G., Oliveira, \& Leite, M. B. F. (2003). The epidemiological models SI with a fuzzy transmission. Computer and Mathematics with Applications 45, 1619-1628.

https://doi.org/10.1016/S08981221(03)00141-X

Castillo-Garsow, C., Jordan-Salivia, G., \& Herrera, A. R. (1997). Mathematical models for the dynamics of tobacco use, recovery, and relapse. Technical Report Series Cornel University, BU1505-M.

Gunawan, A. Y., \& Nurtamam, M. E. (2009). "A Simple Dynamical Model for the Growth of Smoker Population." Journal of the Indonesian Mathematical Society, 14(1),63-72. http://dx.doi.org/10.22342/jims.14.1.8 $\underline{2.63-7}$

Massad, E. et al. (2008): Fuzzy Logic in Action: Aplication in Epidemiology and beyond. Berlin: Springer.

World Health Organization. 10 facts on the global tobacco epidemic. Retrieved October 1, 2020, Retrieved from http://www.who.int/features/factfiles tobacco epidemic/tobacco epidemic facts/en/.

Zadeh, L.A. (1965). Fuzzy sets. Information and Control 8(3), 338-353. https://doi.org/10.1016/S00199958(65)90241-X

Zainab, A., Sarah, A., \& Salma, A. 2014. Global Dynamics of a Mathematical Model on Smoking. International Scholarly Research Notice, Vol. 2014, Article ID 847075, 7 pages. https://doi.org/10.1155/2014/847075 